\title{
Vascular endothelial growth factor correlation with clinicopathological parameters in ovarian cancer
}

\author{
Mahy Egiz*, Alaa Masoud, Hamed Ellakwa, Mohamed Adel Elsayed
}

Department of Obstetrics and Gynecology, Menoufia University, Graduate School of Medicine, Menoufia, Egypt

Received: 09 March 2019

Accepted: 19 March 2019

\section{*Correspondence:}

Dr. Mahy Egiz,

E-mail: mahyegiz@yahoo.com

Copyright: () the author(s), publisher and licensee Medip Academy. This is an open-access article distributed under the terms of the Creative Commons Attribution Non-Commercial License, which permits unrestricted non-commercial use, distribution, and reproduction in any medium, provided the original work is properly cited.

\begin{abstract}
Epithelial ovarian cancer (EOC) is one of the most lethal gynaecologic malignancies with an increasing incidence worldwide; there is an increasing need for the identification of novel prognostic biomarkers in EOC patients. Given the key role of angiogenesis and growth factors in the biology of tumorigenesis, vascular endothelial growth factor (VEGF) is considered a milestone in the process of ovarian cancer progression and invasiveness. Authors aimed in the present study to evaluate the relevance of serum level of VEGF with clinicopathological parameters in patients with EOC. VEGF is reported to be correlated with variable parameters in EOC patients including International Federation of Gynecology and Obstetrics (FIGO) classification, lymph nodal involvement and ascites formation. In the following review, authors discussed these correlations and distinguished the possible future role of VEGF as a promising prognostic biomarker for EOC patients.
\end{abstract}

Keywords: Angiogenesis, Epithelial ovarian cancer, Ovarian cancer, Vascular endothelial growth factor

\section{INTRODUCTION}

Epithelial ovarian cancer (EOC) is the most lethal gynecologic malignancy and the fifth most common cause of cancer-related death in women. ${ }^{1}$ Cancer has the ability to spread to adjacent or distant organs resulting in life-threatening conditions. ${ }^{2}$ For the metastatic spread of cancer tissue, the growth of the vascular network is important which is known by angiogenesis and lymphangiogenesis. ${ }^{3}$

Angiogenic and lymphangiogenic factors are increasingly receiving attention, especially in the field of neoplastic vascularization. ${ }^{4}$ Angiogenesis is regulated by both activator and inhibitor molecules. More than a dozen different proteins have been identified. Levels of expression of angiogenic factors reflect the aggressiveness of tumor cells. ${ }^{5,6}$ Among these, the vascular endothelial growth factor (VEGF) family and its receptors represent a key pathway. High levels of VEGF in serum and ascites, and high VEGF expression on ovarian carcinoma tumors, have been associated with ovarian tumor progression and poor prognosis., ${ }^{3,-11}$

Authors aimed in the present review to discuss the relevance of serum levels of VEGF in patients with EOC with clinicopathological characteristics. Therefore, VEGF can be a promising prognostic biomarker for EOC patients.

\section{OVARIAN CANCER}

Epithelial ovarian cancer (EOC) is the most lethal gynecologic malignancy and the fifth most common cause of cancer-related death in women. The estimated annual incidence of this disease worldwide is just over 200,000 individuals, with approximately 125,000 deaths. ${ }^{1}$ Ovarian cancer is often called 'silent cancer' because of 
its diffuse symptoms. The lethality of ovarian carcinoma primarily stems from the inability to detect the disease at an early, organ-confined stage, and the lack of effective therapies for advanced-stage metastasizing disease. ${ }^{12}$

Unlike cervical cancer, ovarian cancer does not have a detectable pre-invasive phase, and little evidence exists for progression from stage I to stage III disease. Therefore, to date, there is no effective or recommended way to screen for ovarian cancer in the general population. ${ }^{13}$ Recent studies proposed Vascular Endothelial Growth Factor (VEGF) as a prognostic biomarker in ovarian cancer revealing positive expression of VEGF at a significantly higher frequency in ovarian tumor tissue. ${ }^{3}$ These data suggest a promising future role of VEGF as a biomarker in ovarian cancer.

\section{THE RISK OF OVARIAN CANCER}

Unfortunately, identifying high-risk women can be difficult because ovarian cancer risk factors are not well understood. If high-risk women can be identified, riskreducing surgery can be performed, and ovarian cancer can be prevented. Personal risk factors include age, a personal history of nulliparity, oral contraceptive use, obesity, breast cancer, and menopausal status. Increased parity decreases the risk by $50 \%$ over nulliparity, as does oral contraceptive use for 5 years. ${ }^{2}$ Various studies have demonstrated the association between obesity and ovarian cancer. Overall, it seems that obese women (those with a body mass index of at least 30) have a higher risk of developing ovarian cancer. ${ }^{14}$ Women with a BRCA1 or BRCA2 mutation who have been diagnosed with and treated for breast cancer continue to have a high risk of developing ovarian cancer. Therefore, prophylactic measures for the prevention of ovarian cancer should be considered, especially for women with stage I breast cancer. ${ }^{15}$ The family history of ovarian cancer also increases a woman's lifetime risk of developing this cancer, in addition, some other types of cancer such as colorectal and breast cancer is linked to an increased risk of ovarian cancer. This is because these cancers can be caused by an inherited mutation (change) in certain genes that cause a family cancer syndrome that increases the risk of ovarian cancer. ${ }^{16}$

\section{OVARIAN CANCER METASTATIC BEHAVIOR}

The biological behavior of ovarian carcinoma is unique, differing markedly from the classic and well-studied pattern of hematogenous metastasis found in most other cancers. ${ }^{17}$ The task of metastasis appears to be easier for ovarian carcinoma. Once the cancer cells have detached as single cells or clusters, they metastasize through a passive mechanism, carried by the physiological movement of peritoneal fluid to the peritoneum and omentum. ${ }^{4}$ Many previous reports confirmed that Paget's "seed and soil" theory holds true for ovarian carcinoma. The "soil" for ovarian carcinoma is the mesothelium that covers all organs within the peritoneal cavity, including the omentum and the diaphragm. ${ }^{18}$ Before the ovarian carcinoma cells detach and start their metastatic journey, they often undergo an epithelial-to-mesenchymal transition (EMT), which eases the attachment of epithelial cells to the basement membrane and loosens the intercellular adhesions between the cancer cells. Signals inducing EMT in cancer progression are multiple and diverse including growth factors, such as epidermal growth factor (EGF) and fibroblast growth factor (FGF), platelet-derived growth factor (PDGF), insulin-like growth factor (IGF) and vascular endothelial growth factor (VEGF). ${ }^{19}$

\section{ANGIOGENESIS IN OVARIAN CANCER}

It is well established that angiogenesis, the development of new blood vessels from the existing vasculature, is crucial for the growth and metastatic spread of solid tumors beyond 100-200 $\mu$ mas diffusion alone can no longer provide the nutrients required for the growing tumor. ${ }^{6}$ Thus, upregulation of angiogenesis is a key step in sustained tumor growth and also is critical for tumor metastasis. Angiogenesis is regulated by both activator and inhibitor molecules. Early during tumor progression, an angiogenic switch occurs disturbing the physiological balance that keeps the adult vasculature quiescent towards new vessel formation. This proangiogenic state is characterized by upregulation of factors that stimulate vasculogenesis and down regulation of antiangiogenic proteins. Among these, the VEGF family and its receptors represent a key pathway. ${ }^{5}$

\section{VEGF AS AN ANGIOGENIC FACTOR IN OVARIAN CANCER}

There are seven family members (VEGF A-E, placental growth factor [P1GF-1 and PlGF-2), and multiple isoforms of VEGF-A result from alternative mRNA splicing. This family of growth factors signals through cell surface tyrosine kinase receptors (VEGFR-1 [fmslike tyrosine kinase-1; FLT-1], VEGFR-2 [KDR/Flk-1], and VEGFR-3 [Flt-3]) expressed on normal endothelial cells. Preferential binding to receptors has been observed, with VEGF-A binding specifically to VEGFR-1 and -2, VEGF-B and PIGF-1 and -2 binding to VEGFR1, and VEGF-C and -D activating VEGFR-3. ${ }^{8}$ The role of VEGF-EGFR axis in ovarian cancer has been documented in many studies, coming to further prominence since the recent recognition that anti-VEGF therapies exert clinical efficacy in women with ovarian cancer. VEGF is detectable by immunostaining in most ovarian tumors and omental metastases and is measurable in the ascites fluid and serum of patients with ovarian cancer. $^{3,10}$

\section{VEGF BIOLOGICAL BEHAVIOR IN OVARIAN CANCER}

It has been noted that the expression of VEGF and VEGFR in ovarian cancer is an independent poor 
prognostic factor. Importantly, VEGF, VEGFR-1, and VEGFR-2 expressions are cancer-specific, with strong expression being noted in malignant tumors, intermediate expression in borderline tumors, and weak or no staining in normal ovarian tissue. ${ }^{10}$ Other members of the VEGF signaling pathways are expressed in ovarian tumors, including neuropilin and VEGFR-3 that is implicated in lymphangiogenesis and ascites formation. ${ }^{20}$ Aside from promoting angiogenesis, through direct stimulation of endothelial cells, VEGF exerts other important functions in OC. By augmenting the permeability of the microvasculature, VEGF enhances the extravasation of plasma proteins and water in the tumor niche; this contributes to the reshaping of the extracellular matrix that becomes favorable to cell migration and proliferation. The stroma is thus invaded by endothelial cells, which will form new blood vessels, and by fibroblasts, which synthesize proteoglycans and glycosaminoglycans, contributing to its maturation. ${ }^{21}$ In addition, increased capillary permeability induced by VEGF causes ascites formation. This has been demonstrated in OC xenograft models that harbor high VEGF concentrations in malignant ascites associated with tumors. It is not surprising therefore those VEGF inhibitors potently inhibit ascites production and reduce intraperitoneal tumor growth. ${ }^{22}$

The newly formed, leaky capillaries induced by VEGF are favorable to metastasis as their basement membranes are fenestrated and allow tumor cells to disseminate. ${ }^{6}$ Through its effects on vasculature, stroma and tumor cells, the VEGF-VEGFR axis orchestrates in OC significant pro-tumorigenic and metastatic effects.

\section{VEGF AS A PROGNOSTIC BIOMARKER IN OVARIAN CANCER}

High levels of VEGF in serum and ascites, and high VEGF expression on ovarian carcinoma tumors have been associated with ovarian tumor progression and poor prognosis. Inhibition of human VEGFR2 in an ovarian cancer xenograft model inhibited tumor growth by blocking the VEGFR2 on the human tumor cells. Several VEGF/VEGFR inhibitory agents are in clinical testing and early results are promising suggesting that the regrowth of disease is delayed if VEGF is depleted from the tumor for a prolonged period of time. ${ }^{23}$

In addition, these markers could predict response to antiangiogenic therapy including measurement of proangiogenic protein concentration in plasma or urine (e.g., VEGF, PDGF, PlGF, thrombospondin 1[TSP1]). ${ }^{24}$

Serum biomarkers are attractive targets for early detection protocols and CA125 has been widely used in screening trials. CA125 has limitations of specificity for ovarian cancer as an elevation in serum can also occur in pregnancy, endometriosis, and menstruation. ${ }^{25}$ Panels of biomarkers are thought to offer the potential for higher discriminatory power. However, recent studies which constructed putative biomarker panels with samples from the PLCO cancer trial found no improvement in diagnostic power in pre-clinical samples, leaving CA125 as the single best biomarker for ovarian cancer screening. ${ }^{26}$ To improve outcomes for ovarian cancer patients there is a necessity to identify new biomarkers that are capable of improving on or complementing CA125 for early detection of ovarian cancer. ${ }^{27}$

\section{VEGF CORRELATION WITH CLINICOPATHOLOGICAL PARAMETERS IN EOC}

\section{Lymph nodal involvement}

Lymph node involvement in gynecologic neoplasms implies a worse prognosis and changes in the therapeutic approach. Generally, ovarian cancer has three routes for lymphatic metastasis. Firstly, through lymphatic vessels within the infundibulopelvic ligament towards the paraaortic and para-caval lymph nodes. Once the pelvic and para-aortic lymph nodes have been involved, lymphatic channels within the diaphragm and retro-peritoneum will facilitate dissemination above the diaphragm. Less commonly, the second route follows the sub-ovarian plexus in the bilateral broad ligament towards the obturator and pelvic iliac lymph nodes. The third potential route follows the bilateral round ligament of the uterus to the external iliac and deep inguinal lymph nodes. $^{28}$

Involvement of pelvic and para-aortic lymph nodes is common, particularly in advanced-stage disease (stages III and IV), with an incidence of $40 \%-88 \%$. Systematic lymphadenectomy as part of the surgical staging of apparent early-stage ovarian cancer is associated with a statistically significant increase in median operative time, median blood loss, and the proportion of patients undergoing blood transfusions. However, significantly improves progression-free survival (PFS) rates, without a statistically significant impact on overall survival (OS). ${ }^{29}$

Lymphatic spread of early-stage ovarian cancer upstages the patient to FIGO stage III. Therefore, lymphadenectomy in ovarian cancer patients provides an essential tool for ovarian cancer staging, a way for tailored management strategies and also offers greater survival rates for these patients. However, systemic lymphadenectomy may increase surgical morbidity and postoperative complications, thus, it is mandatory to investigate possible biomarkers for prediction of lymph node involvement in ovarian cancer. ${ }^{30}$

Li et al, reported that patients with metastasis to the peritoneum and lymph nodes had higher serum VEGF levels than patients without metastasis, which is in agreement with a former investigation recorded that VEGF expression was correlated with broad metastasis in ovarian cancer. ${ }^{9,31}$ Accordingly, routine follow-up with repeated measurement of serum levels of VEGF and 
ovary ultrasonography may facilitate detection of small tumors and early tumor metastasis, which might improve the life expectancy of ovarian cancer patients. Additionally, from a biological viewpoint, VEGF expression is related to the malignant potential of tumors and the tendency of tumors to metastasize.

\section{FIGO stage}

A correlation between VEGF expression and the surgical stage was recorded, with advanced stage tumors demonstrating stronger VEGF expression than earlystage tumors. ${ }^{32}$

The prevailing, recently modified International Federation of Gynecology and Obstetrics (FIGO) classification scheme includes a revision of stage III: Classification of ovarian cancers as stage IIIA1 is based on spread to the retroperitoneal lymph nodes without intraperitoneal dissemination, since an analysis of patients with these findings indicated that their survival rate is significantly better than that of patients with intraperitoneal dissemination. Thus, the overall survival rate for ovarian cancer patients with retroperitoneal lymph node involvement but no peritoneal carcinomatosis is $58 \%-84 \%$, compared with $18 \%-36 \%$ for patients with retroperitoneal involvement and peritoneal carcinomatosis. However, less than $10 \%$ of ovarian carcinomas spread by means of retroperitoneal lymph node involvement alone (stage IIIA1). ${ }^{33}$

VEGF has been demonstrated to be related to clinical stages and to malignant grades in human tumors. Shen et al, found that VEGF was expressed differently at different stages in ovarian cancer. There was no difference in positive rate between early stage and late stage $(94.6 \%, 35 / 37$ versus $100 \%, 27 / 27)$. However, $32.4 \%$ in the former group were strongly-positive, compared with $70.4 \%$ strongly-positive in the latter group (p500 ml), or high malignant potential in human ovarian cancer. $^{34}$

\section{Histopathological type}

The common histological types of epithelial ovarian carcinoma are a serous, endometrioid, clear cell, and mucinous carcinoma, as well as carcinomas of mixed type. These distinct morphological entities are growingly perceived to be tumors of different etiology, with unique genetic and phenotypic characteristics and different clinical behavior, including response to chemotherapy. Clinical observations and genetic studies have divided ovarian cancer into two major subtypes. ${ }^{35}$ Type 1 cancers are composed of low-grade serous cancers, endometrioid and clear cell cancers, and mucinous cancers. This group tends to grow locally, metastasize late, and behave in a more indolent fashion. Type 2 cancers are composed of high-grade serous cancers, carcinosarcomas, and undifferentiated carcinomas. These are highly aggressive malignancies that generally present at an advanced stage.
In addition to clinical differences, there are also notable genetic differences. Type 1 cancers are associated with mutations in KRAS, ARID1A, PIK3CA, PTEN, and BRAF; whereas the majority of type 2 cancers are associated with mutations in TP53. ${ }^{36}$

Mukherjee et al, determined the VEGF expression by immunohistochemistry. ${ }^{10}$ Among the 22 malignant cases, $18(81.8 \%)$ were high expressers, only $3(13.6 \%)$ were low expressers and expression was absent in one case. 11 cases out of 14 of high grade serous papillary cystadenocarcinoma, all five cases of mucinous cystadenocarcinoma and the two cases of endometrioid carcinoma showed high VEGF expression. VEGF expression was absent in clear cell carcinoma, This finding is comparable to the study of Premalata et al, with 26 of $78(33.3 \%)$ cases of primary malignant epithelial ovarian neoplasm showing high VEGF expression. ${ }^{11}$

\section{Age at diagnosis}

As with most cancers, the risk of developing ovarian cancer increases as a woman gets older. Women over the age of 50 have a higher risk, and most cases of ovarian cancer occur in women who have already gone through the menopause. More than half the cases of ovarian cancer diagnosed are women over 65 years. Early age at menarche and late age at menopause exhibit a moderate increase in ovarian cancer risk. ${ }^{37}$ Although not a very commonly studied parameter, it seems that age is also associated with the possibility of the apparition of lymph node metastases. It has been demonstrated that younger age at diagnosis is usually associated with a poorer outcome and, with a higher rate of positive para-aortic lymph nodes, especially due to a higher biological aggressiveness. ${ }^{38}$

Duncan et al, did not find any association between VEGF expression and patients' age. ${ }^{32}$ Similar results are reported by Siddiqui et al, consistent with these results, Ranjbar et al, revealed no association between VEGF expression and age, among the 48 patients with serous ovarian carcinoma with high VEGF expression, 25 $(52.3 \%)$ were $\leq 53$ years old, and $23(47.7 \%)$ were $<53$ years old..$^{3,39}$

The extent of metastasis in ovarian cancer patients and sizes of tumor residue after the operation. As previously observed, the serum VEGF levels were significantly higher in the ovarian cancer patients with metastasis to lymph nodes or omentum than in the ovarian cancer patients without lymph node or omentum metastasis $(\mathrm{p}<0.05)$. In addition, the serum VEGF levels were higher $(\mathrm{p}<0.05)$ in patients with residual tumor sizes $>2$ $\mathrm{cm}$ than in patients with residual tumor sizes $\leq 2 \mathrm{~cm}$ after surgical therapy. ${ }^{9}$

VEGF expression was correlated with broad metastasis in ovarian carcinoma. ${ }^{31}$ Accordingly, the combined evaluation of serum levels of VEGF and ovary 
ultrasonography may facilitate detection of small tumors and early tumor metastasis, which might improve the life expectancy of ovarian cancer patients. In addition, the levels of serum VEGF reported being strikingly lower after surgery than those before therapy, the level of serum VEGF after operation depends on the residual size of the tumor.

\section{Ascites and VEGF level}

VEGF is secreted in malignant ascites at concentrations 50-200 times higher than in non-malignant fluid and plays an important role in ascites formation in ovarian cancer patients via increasing peritoneal permeability by down regulation of Claudin $5 .^{7}$ VEGF is a specific endothelial mitogen, the major factor for a vascular generation, which plays an important role in tumorous vessels generation and ascites production by facilitating tumor vascular endothelial proliferation and elevating local capillary permeability. ${ }^{40}$ Higher levels of serum VEGF were reported with more ascites (>500ml). Reciprocally, markedly lower levels of serum VEGF were measured in patients with fewer ascites $(<500 \mathrm{ml})$, suggesting a strong correlation between VEGF serum levels and ascites in ovarian cancer patients. ${ }^{9}$

\section{ACKNOWLEDGMENTS}

Authors would like to express their appreciation to the Ministry of Higher Education and External Affairs Section for allowing joint supervision program to finish the study in Japan, which allowed the experience to be raised and to conduct a good collaboration between Egypt and Japan.

\section{Funding: No funding sources}

Conflict of interest: None declared

Ethical approval: Not required

\section{REFERENCES}

1. Siegel RL, Miller KD, Jemal A. Cancer statistics, 2018. CA Cancer J Clin. 2018;68(1):7-30.

2. Schildkraut JM, Calingaert B, Marchbanks PA, Moorman PG, Rodriguez GC. Impact of progestin and estrogen potency in oral contraceptives on ovarian cancer risk. J National Cancer Institute. 2002;94(1):32-8.

3. Ranjbar R, Nejatollahi F, Nedaei Ahmadi AS, Hafezi $\mathrm{H}$, Safaie A. Expression of vascular endothelial growth factor (VEGF) and epidermal growth factor receptor (EGFR) in patients with serous ovarian carcinoma and their clinical significance. Iranian $\mathbf{J}$ Cancer Prev. 2015;8(4):e3428.

4. Tarin D, Price JE, Kettlewell MG, Souter RG, Vass AC, Crossley B. Mechanisms of human tumor metastasis studied in patients with peritoneovenous shunts. Cancer research. 1984;44(8):3584-92.
5. Dimova I, Popivanov G, Djonov V. Angiogenesis in cancer-general pathways and their therapeutic implications. J BUON. 2014;19(1):15-21.

6. Folkman J. Angiogenesis in cancer, vascular, rheumatoid and other disease. Nat Med. 1995;1(1):27-31.

7. Herr D, Sallmann A, Bekes I, Konrad R, Holzheu I, Kreienberg R, et al. VEGF induces ascites in ovarian cancer patients via increasing peritoneal permeability by downregulation of Claudin 5. Gynecol Oncol. 2012;127(1):210-6.

8. Leppanen VM, Prota AE, Jeltsch M, Anisimov A, Kalkkinen N, Strandin T, et al. Structural determinants of growth factor binding and specificity by VEGF receptor 2. Proc Natl Acad Sci U S A. 2010;107(6):2425-30.

9. Li L, Wang L, Zhang W, Tang B, Zhang J, Song H, et al. Correlation of serum VEGF levels with clinical stage, therapy efficacy, tumor metastasis and patient survival in ovarian cancer. Anticancer Res. 2004;24(3b):1973-9.

10. Mukherjee S, Pal M, Mukhopadhyay S, Das I, Hazra $\mathrm{R}$, Ghosh S, et al. vegf expression to support targeted therapy in ovarian surface epithelial neoplasms. JCDR. 2017;11(4):EC43-6.

11. Premalata CS, Umadevi K, Shobha K, Anurekha M, Krishnamoorthy L. Expression of VEGF-A in epithelial ovarian cancer: correlation with morphologic types, grade and clinical stage. Gulf $\mathbf{J}$ Oncol. 2016;1(21):49-54.

12. Bankhead CR, Collins C, Stokes-Lampard H, Rose $\mathrm{P}$, Wilson S, Clements A, et al. Identifying symptoms of ovarian cancer: a qualitative and quantitative study. BJOG. 2008;115(8):1008-14.

13. Buys SS, Partridge E, Black A, Johnson CC, Lamerato L, Isaacs C, et al. Effect of screening on ovarian cancer mortality: the Prostate, Lung, Colorectal and Ovarian (PLCO) Cancer Screening Randomized Controlled Trial. JAMA. 2011;305(22):2295-303.

14. Foong KW, Bolton H. Obesity and ovarian cancer risk: A systematic review. Post Reproductive Health. 2017;23(4):183-98.

15. Metcalfe KA, Lynch HT, Ghadirian P, Tung N, Olivotto IA, Foulkes WD, et al. The risk of ovarian cancer after breast cancer in BRCA1 and BRCA2 carriers. Gynecol Oncol. 2005;96(1):222-6.

16. Chui MH, Ryan P, Radigan J, Ferguson SE, Pollett A, Aronson M, et al. The histomorphology of Lynch syndrome-associated ovarian carcinomas: toward a subtype-specific screening strategy. Am J Surg Pathol. 2014;38(9):1173-81.

17. Gupta GP, Massague J. Cancer metastasis: building a framework. Cell. 2006;127(4):679-95.

18. McGee SF, Lanigan F, Gilligan E, Groner B. Mammary gland biology and breast cancer. Conference on Common Molecular Mechanisms of Mammary Gland Development and Breast Cancer Progression. EMBO Reports. 2006;7(11):1084-8. 
19. Huber MA, Kraut N, Beug H. Molecular requirements for epithelial-mesenchymal transition during tumor progression. Current Opinion in Cell Biology. 2005;17(5):548-58.

20. Jeon BH, Jang C, Han J, Kataru RP, Piao L, Jung K, et al. Profound but dysfunctional lymphangiogenesis via vascular endothelial growth factor ligands from $\mathrm{CD} 11 \mathrm{~b}+$ macrophages in advanced ovarian cancer. Cancer Res. 2008;68(4):1100-9.

21. Matkar PN, Ariyagunarajah R, Leong-Poi H, Singh KK. Friends turned foes: angiogenic growth factors beyond angiogenesis. Biomolecules. 2017;7(4).

22. Byrne AT, Ross L, Holash J, Nakanishi M, Hu L, Hofmann JI, et al. Vascular endothelial growth factor-trap decreases tumor burden, inhibits ascites, and causes dramatic vascular remodeling in an ovarian cancer model. Clinical Cancer Research: J Am Assoc Cancer Res. 2003;9(15):5721-8.

23. Garcia AA, Hirte H, Fleming G, Yang D, Tsao-Wei DD, Roman L, et al. Phase II clinical trial of bevacizumab and low-dose metronomic oral cyclophosphamide in recurrent ovarian cancer: a trial of the California, Chicago, and Princess Margaret Hospital phase II consortia. J Clin Oncol: J Am Soc Clin Oncol. 2008;26(1):76-82.

24. Willett CG, Duda DG, Di Tomaso E, Boucher Y, Ancukiewicz M, Sahani DV, et al. Efficacy, safety, and biomarkers of neoadjuvant bevacizumab, radiation therapy, and fluorouracil in rectal cancer: a multidisciplinary phase II study. J Clin Oncol: J the Am Soc Clin Oncol. 2009;27(18):3020-6.

25. Vlasova MA, Moshkovskii SA, Safarova MP, Makarov OV, Archakov AI. Molecular diagnostics of ovarian cancer using proteome techniques. Biomeditsinskaia Khimiia. 2005;51(4):367-83.

26. Cramer DW, Bast RC, Berg CD, Diamandis EP, Godwin AK, Hartge $P$, et al. Ovarian cancer biomarker performance in prostate, lung, colorectal, and ovarian cancer screening trial specimens. Cancer Prev Res. 2011;4(3):365-74.

27. Maringe C, Walters S, Butler J, Coleman MP, Hacker N, Hanna L, et al. Stage at diagnosis and ovarian cancer survival: evidence from the International Cancer Benchmarking Partnership. Gynecol Oncol. 2012;127(1):75-82.

28. Watanabe Y, Satou T, Nakai H, Etoh T, Dote K, Fujinami N, et al. Evaluation of parametrial spread in endometrial carcinoma. Obstet Gynecol. 2010;116(5):1027-34.

29. Panici PB, Maggioni A, Hacker N, Landoni F, Ackermann S, Campagnutta E, et al. Systematic aortic and pelvic lymphadenectomy versus resection of bulky nodes only in optimally debulked advanced ovarian cancer: a randomized clinical trial. J Nat Cancer Inst. 2005;97(8):560-6.
30. Achouri A, Huchon C, Bats AS, Bensaid C, Nos C, Lecuru F. Complications of lymphadenectomy for gynecologic cancer. Europ J Surg Oncol: J Europ Soc Surg Oncol Brit Assoc Surg Oncol. 2013;39(1):81-6.

31. Duyndam MC, Hilhorst MC, Schlüper HM, Verheul HM, Van Diest PJ, Kraal G, et al. Vascular endothelial growth factor-165 overexpression stimulates angiogenesis and induces cyst formation and macrophage infiltration in human ovarian cancer xenografts. Am J Pathol. 2002;160(2):537-48.

32. Duncan TJ, Al-Attar A, Rolland P, Scott IV, Deen S, Liu DT, et al. Vascular endothelial growth factor expression in ovarian cancer: a model for targeted use of novel therapies? Clin Cancer Res: J American Assoc Can Res. 2008;14(10):3030-5.

33. Berek JS. Lymph node-positive stage IIIC ovarian cancer: a separate entity? Int J Gynecol Cancer: J Int Gynecol Cancer Soc. 2009;19 Suppl 2:S18-20.

34. Shen GH, Ghazizadeh M, Kawanami O, Shimizu H, Jin E, Araki T, et al. Prognostic significance of vascular endothelial growth factor expression in human ovarian carcinoma. Brit J Cancer. 2000;83(2):196-203.

35. Kurman RJ, Shih Ie M. The origin and pathogenesis of epithelial ovarian cancer: a proposed unifying theory. Am J Surg Pathol. 2010;34(3):433-43.

36. Kurman RJ, Shih Ie M. Molecular pathogenesis and extraovarian origin of epithelial ovarian cancer-shifting the paradigm. Hum Pathol. 2011;42(7):91831.

37. Schildkraut JM, Cooper GS, Halabi S, Calingaert B, Hartge P, Whittemore AS. Age at natural menopause and the risk of epithelial ovarian cancer. Obstet Gynecol. 2001;98(1):85-90.

38. Ayhan A, Gultekin M, Taskiran C, Celik NY, Usubutun A, Kucukali T, et al. Lymphatic metastasis in epithelial ovarian carcinoma with respect to clinicopathological variables. Gynecol Oncol. 2005;97(2):400-4.

39. Siddiqui GK, Elmasry K, Wong AT, Perrett C, Morris R, Crow JC, et al. Prognostic significance of intratumoral vascular endothelial growth factor as a marker of tumour angiogenesis in epithelial ovarian cancer. Europ J Gynaecol Oncol. 2010;31(2):156-9.

40. Fujimoto J, Sakaguchi H, Hirose R, Ichigo S, Tamaya T. Biologic implications of the expression of vascular endothelial growth factor subtypes in ovarian carcinoma. Cancer. 1998;83(12):2528-33.

Cite this article as: Egiz M, Masoud A, Ellakwa H, Elsayed MA. Vascular endothelial growth factor correlation with clinicopathological parameters in ovarian cancer. Int J Reprod Contracept Obstet Gynecol 2019;8:1700-5. 